\title{
Coronary artery vasculitis in a patient of Behçet's disease.
}

\author{
Haiyang $\mathrm{Wei}^{1}$, Zhenqiang $\mathrm{Xu}^{1}$, Bin $\mathrm{Hu}^{1}$, Chengwei Zou ${ }^{2}$, and Gang Zhang ${ }^{1}$ \\ ${ }^{1}$ Shandong Provincial Hospital \\ ${ }^{2}$ Affiliation not available
}

September 25, 2021

\begin{abstract}
We report a case of coronary artery vasculitis in Behçet's disease. The patient was treated with the off-pump coronary artery bypass grafting. This case shows the diagnostic value of coronary computed tomography angiography in coronary artery inflammatory disease.
\end{abstract}

\section{Coronary artery vasculitis in a patient of Behçet's disease}

Haiyang Wei $\mathrm{MM}^{1+}$, Zhenqiang Xu MM${ }^{1+}$, Bin Hu MD ${ }^{1}$, Chengwei Zou MD ${ }^{1}$, Gang Zhang $\mathrm{MD}^{1 *}$

${ }^{1}$ Department of Cardiac Surgery, Shandong Provincial Hospital Affiliated to Shandong First Medical University, Jinan 250021, China

* Corresponding author. Tel: +86 531 68776353, Email: surgeonzg@outlook.com

+ These authors contributed equally to this work.

\section{Corresponding Author:}

Tel: +86 15966603949

Email:surgeonzg@outlook.com

All procedures performed in studies involving human participants were in accordance with the 1964 Helsinki declaration and its later amendments or comparable ethical standards.

Oral informed consent was obtained.

The authors declare that there are no conflict of interests.

No funds supported for this research.

\section{Abstract}

We report a case of coronary artery vasculitis in Behcet's disease. The patient was treated with the off-pump coronary artery bypass grafting. This case shows the diagnostic value of coronary computed tomography angiography in coronary artery inflammatory disease.

A 65-year-old man with more than 20 years of history of Behcet's disease (BD) was admitted to hospital with paroxysmal chest tightness and chest pain. Prior to his admission, he was regularly treated with thalidomide and showed no oral and vulvar ulcers in recent years. His blood lipids are normal. His risk factors for coronary heart disease include smoking ( 5 cigarettes/day for 30 years) and diabetes.

Results of the coronary angiography revealed stenosis in the left main artery (Figure 1A, Supplementary Video S1) and right coronary artery (Figure 1B, Supplementary Video S2). Coronary computed tomography 
angiography (CCTA) showed the vascular wall of the left main artery (Figure 1C) and the right coronary artery stenosis (Figure 1D) was thickened in a diffuse ring, showing the rough vascular wall and the evidently narrowed vascular lumen.

The patient was treated with the off-pump coronary artery bypass grafting with hydrocortisone (100 mg ivdrip) given prior to the operation. After operation, the patient was orally given prednisone $30 \mathrm{mg}$ and thalidomide $25 \mathrm{mg}$ every day. The patient recovered well and showed no discomfort such as chest tightness or chest pain after the normal physical activities.

BD is a type of vasculitis of unknown etiology and is characterized by oral sores, vulvar ulcers, and ocular lesions ${ }^{1}$. The coronary artery disease is rare in patients of BD with an incidence rate of $\sim 0.5 \%$ on the Silk Road. The coronary artery aneurysms are more common than stenosis. We think coronary artery stenosis in this patient is caused by $\mathrm{BD}$, instead of coronary atherosclerotic heart disease. The former is the full layer of blood vessel wall thick leads to stenosis. The latter caused by atherosclerosis plaques at vascular intima, and is mostly manifested on CT as high density. This case shows the diagnostic value of CCTA in coronary artery inflammatory disease.
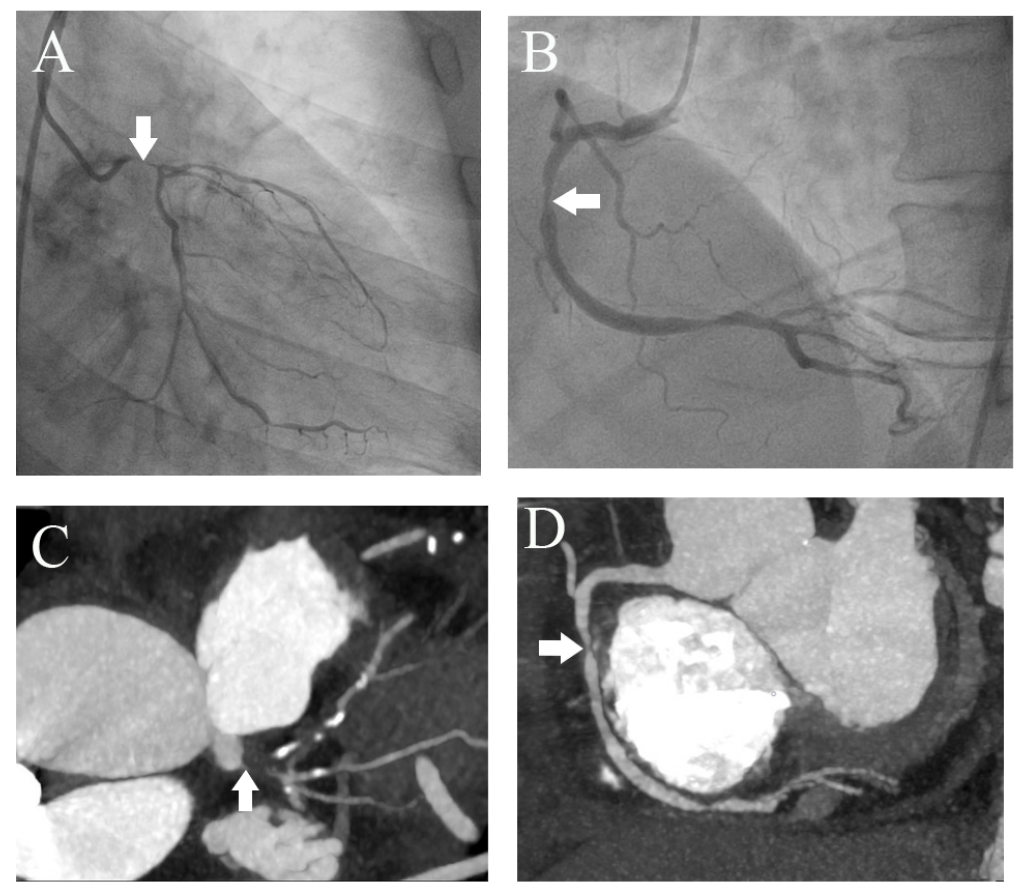

Figure 1. Coronary angiography showing $~ 95 \%$ stenosis (white arrow) in the left main artery (A ) and $\sim 5 \%$ stenosis (white arrow) in the middle and distal part of the right coronary artery (B ). Multidetector computed tomography showing the vascular walls of the left main artery $(\mathbf{C})$ and right coronary artery stenosis (D ) were thickened in a diffuse ring, showing the rough vascular wall and the evidently narrowed vascular lumen.

\section{References}

1. Selami Demirelli, Husnu Degirmenci, Sinan Inci, et al. Cardiac manifestations in Behcet's disease. Intractable Rare Dis Res. 2015 May ;4(2): 70-5.

\section{Hosted file}


Title page.docx available at https://authorea.com/users/365750/articles/539103-coronaryartery-vasculitis-in-a-patient-of-beh\%C3\%A7et-s-disease
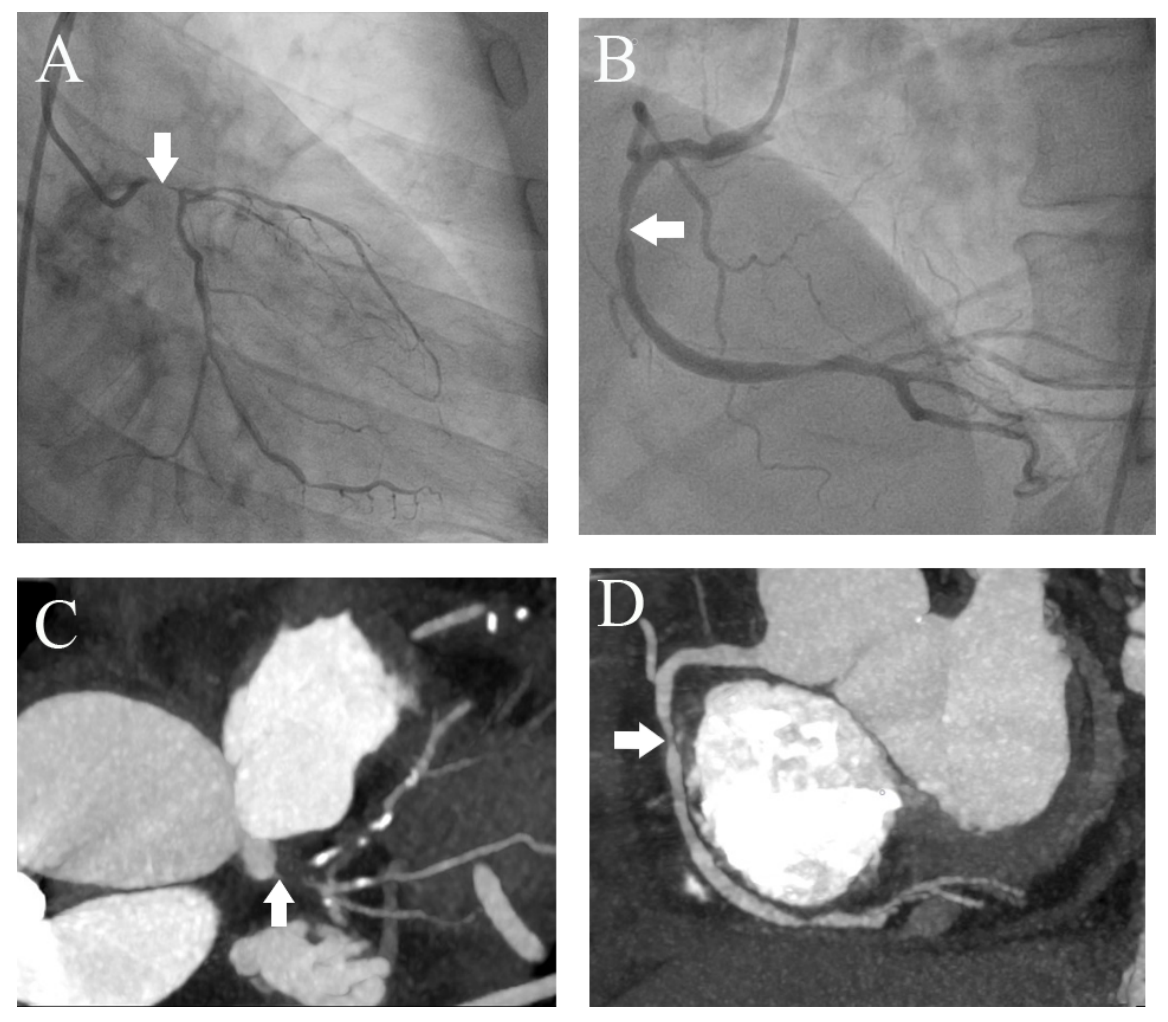